\title{
Effect of Accommodation Training on Visual Function of Visual Inspection Workers and Middle-Aged People
}

\author{
Masumi Takada1, Masaru Miyao ${ }^{2}$, Motohiko Satoh¹, Kazuki Yoshikawa ${ }^{3}$, Yasuyuki Matsuura ${ }^{3}$ and Hiroki Takada ${ }^{1,3 *}$ \\ ${ }^{1}$ Graduate School of Medicine, Aichi University of Medicine, Nagakute 480-1195, Japan \\ ${ }^{2}$ Graduate School of Information Science, Nagoya University, Japan \\ ${ }^{3}$ Graduate Schools of Engineering, University of Fukui, Fukui 910-8507, Japan
}

\begin{abstract}
By relaxing the contracted focus-adjustment muscles around the eyeball, such as the ciliary and extraocular muscles, improvement of the pseudo-myopia is expected. This understanding has led to the accommodation training in which the visual target is given by stereoscopic video clips. In this study, we verify short-term effects of the apparatus on eyesight of visual inspection workers (22 females) suffering from eye fatigue and 12 middle-aged persons. In the Measurement 1, the workers were trained in 3 days. Moreover, the middle-aged were investigated on several trials of the eyesight recovering apparatus in the Measurement 2 . As a result, the visual acuity was statistically improved by continuous accommodation training which will promote a ciliary muscle-stretching effect.
\end{abstract}

Keywords: Myopia; Presbyopia; Spherical diopter (SPH); Visual acuity; Stereoscopic images; Ciliary muscle; Accommodation

\section{Introduction}

With the development of computers and widespread use of the internet, near visual tasks, such as Visual Display Terminal (VDT) activities have increased in young to elderly persons, causing social problems. Near visual tasks for a prolonged time strain the ciliary muscle, which may cause abnormalities in the accommodative function of the lens. This condition is called pseudomyopia, considered to be a part of refractive myopia. Prolonged near visual tasks have been reported to possibly induce cervico-omo-brachial syndrome and psychoneurotic symptoms [1]. The main cause of these vision problems is an accommodative function error. Therefore, we assume that it is possible to improve an abnormal accommodative function of the lens by activating the muscles by alternately repeating negative and positive accommodation. By improving the abnormal accommodative function, we can improve or prevent these vision problems. We call this operation "accommodation training." In Japan, an apparatus called MD-SS was developed [2]. This apparatus works by using a Landolt ring drawn on a flat plate that moves back and forth over a distance of $2 \mathrm{~m}$ in order to encourage alternately repeating negative and positive accommodation in the observers. However, the moving distance of the target object is very short. Therefore, the back-and-forth motion of the objects might have no effect on the observers. In order to solve the above mentioned problems, we suggest that the accommodation training is accomplished by gazing at an image in 3D movies, which simulate the back-andforth motion in a stereoscopic space by using a computer and a Liquid Crystal Display (LCD).

For pseudomyopia, stretching exercise of the ciliary muscle, involved in accommodation of the lens, by alternately repeating negative and positive accommodation alleviates strain of the ciliary muscle. Miyao et al. experimentally showed that the lens was accommodated by following stereoscopic images when gazing at them displayed on a CRT or LCD $[3,4]$.

Presbyopia represents senile impairment of near vision due to a reduced accommodative function of the lens with aging. The major cause of the reduction of accommodative function is thickening of the lens with aging, increasing the radius of the frontal curvature of the lens. In addition to this increase in lens volume, the elasticity of the lens capsule decreases, which results in an insufficient increase in the lens curvature even when the ciliary zonule is relaxed by ciliary muscle contraction, reducing the amplitude of accommodation [5]?
Stereoscopic videos utilizing binocular stereoscopic vision often cause unpleasant symptoms of asthenopia, such as headache and vomiting, depending on the audiovisual condition [6]. Ataxia in simulator-induced sickness has been reported. The influence of videoinduced motion sickness on the body has been measured employing subjective scales, such as the Simulator Sickness Questionnaire (SSQ) [7], and by quantitatively investigating the relationship between external factors and internal conditions using physiological indices [8-11], such as respiratory function, electrocardiogram, skin electrical activity, fluctuation of the center of gravity, and electrogastrogram.

A new 3D video construction method has recently been developed to prevent video-induced motion sickness [12,13]. Humans perceive 3-dimensional objects by simultaneous convergence and accommodation of the lens, but stereoscopic videos generally consist of unnatural images perceived along a fixed visual line, negating such convergence and accommodation. Stereoscopic images using the POWER3D method (Olympus Visual Communications Co., Ltd.) prepared in order to reduce inconsistency between experience and the actual senses [14]. Some preceding studies showed that the degree of sickness was reduced by viewing stereoscopic videos prepared using this method $[15,16]$. We focused on stereoscopic videos prepared using this method. An LCD displaying the stereoscopic videos and visual acuity recovery device utilizing liquid crystal shutter eyeglasses (DR.REX Eye Care Program [14]) include several stereoscopic video contents (Figure 1), which induce near and distant visual conditions. The alternating presentation of these with appropriate intervals is expected to improve and prevent myopia and presbyopia.

In this study, we investigated the short-term effect of the accommodation training with the device utilizing stereoscopic videos

*Corresponding author: Hiroki TAKADA, Graduate Schools of Engineering University of Fukui, Fukui 910-8507, Japan, Fax: +81-776-27-8420; E-mail: takada@u-fukui.ac.jp

Received April 19, 2012; Accepted May 24, 2012; Published May 28, 2012

Citation: Takada M, Miyao M, Satoh M, Yoshikawa K, Matsuura Y (2012) Effect of Accommodation Training on Visual Function of Visual Inspection Workers and Middle-Aged People. J Sports Med Doping Stud 2:112. doi:10.4172/21610673.1000112

Copyright: @ 2012 Takada M, et al. This is an open-access article distributed under the terms of the Creative Commons Attribution License, which permits unrestricted use, distribution, and reproduction in any medium, provided the original author and source are credited. 

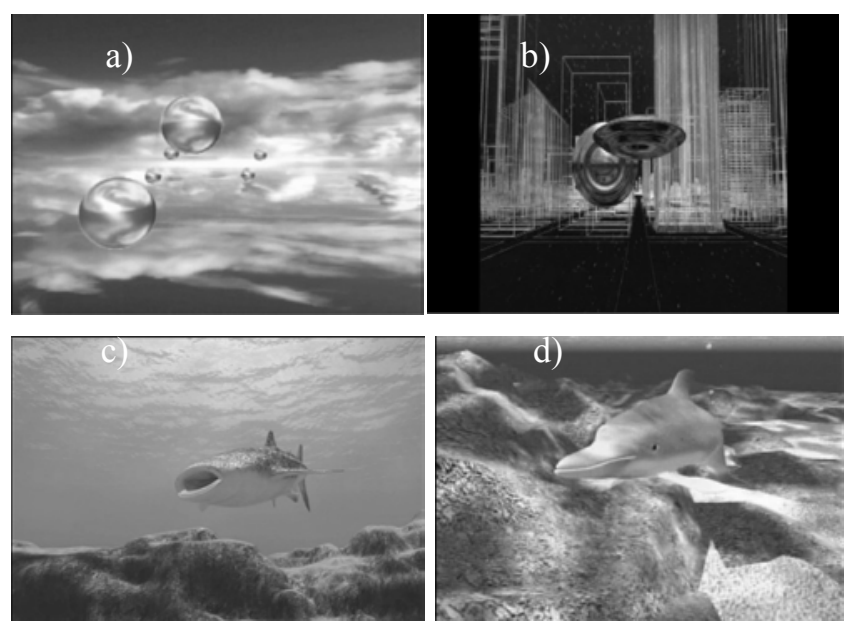

Figure 1: DR.REX Eye Care Program [14] includes several stereoscopic video contents: Sky vision a), Cyber vision b), Under the sea c), Dolphin d).

on visual inspection workers suffering from eye fatigue and middleaged subjects becoming aware of presbyopia.

\section{Material and Method}

The objective and contents of the study and consideration of protection of personal information were explained to all subjects before the experiment, and written informed consent was obtained. The measurement was performed in a dimly lit room (about $260 \mathrm{~lx}$ ) airconditioned at $25^{\circ} \mathrm{C}$. Before (Pre) and after (Post) the accommodation training stated below, the tests:

1) Simulator Sickness Questionnaire (SSQ)

2) Visual Analog Scale (VAS)

3) Objective refractometry

4) Visual acuity test (distant vision)

5) Visual acuity test (near vision)

were performed in this order. The auto visual acuity meter NV-300 (NIDEK) was used for the visual acuity tests that were employed for binocular and monocular visions. Time-course changes in the VAS, the Best Visual Acuity (BVA) at a distance, and that from near were investigated. Findings on Pre and Post values were compared employing the Wilcoxon signed-rank test, setting the significance level to 0.05 .

\section{Measurement 1}

The subjects of the experiment were 22 female visual inspection workers, aged $37 \pm 6$ years. These subjects were also divided into two groups. One group underwent the accommodation training, in which they viewed a stereoscopic video (Figure 1a) for 6 min after the visual inspection work, and the other group was not given any task to perform during the first three consecutive days. Thereafter, the groups switched tasks, and the experiment was performed in a similar manner to collect data without the influence of task order. The above -mentioned items (1)-(5) were performed before the visual inspection work on the first day morning and after the task every experimental day.

\section{Measurement 2}

The subjects were 12 middle-aged persons aged $46.6 \pm 3.5$ years ( 6 males and 6 females). The accommodation training was performed, in which the subjects viewed 4 contents of the DR.REX Eye Care
Program (Figure 1) for 6 minutes each in a random order. Before the accommodation training and after viewing each content, the tests (1)(5) were performed in this order. The subjects then viewed the 4 contents in the same order for 6 minutes each, and the above-mentioned tests (1)-(5) were repeated.

\section{Results}

\section{Measurement 1}

The VAS for the evaluation of the visual fatigue significantly increased after their work (Figure 2a) although the other subjective indices (SSQ sub scores) did not significantly increase.

Variations in the BVA at a distance and from near with the experimental dates are shown in Figure $3 a-4 a$, respectively. The gravitational mean BVA of the 22 subjects increased with the date. Irrespective of the subjects undergoing the accommodation training, when the Wilcoxon signed-rank test was used to compare the BVA before and after the initiation of this experiment, a significant difference in the visual acuity was observed $(\mathrm{p}<0.05)$. The monocular vision test yielded a similar statistical significance. Although in comparison to the values obtained from the previous test, the visual acuity after the visual inspection work increased significantly on the experimental days $(\mathrm{p}<$ 0.05 ), the dioptric mean did not increase throughout this period.

\section{Measurement 2}

Subjective indices did not significantly increase with viewing frequency. The time-course changes in the VAS were shown in Figure $2 \mathrm{~b}$. The degree of asthenopia tended to decrease at $5^{\text {th }}$ and $7^{\text {th }}$ Post viewing than that measured at Pre viewing $(\mathrm{p}<0.10)$.

Changes in the binocular near and distant visual acuities with the frequency of video viewing are shown in Figure 3b. The gravitational mean binocular near and distant visual acuities of the 12 subjects
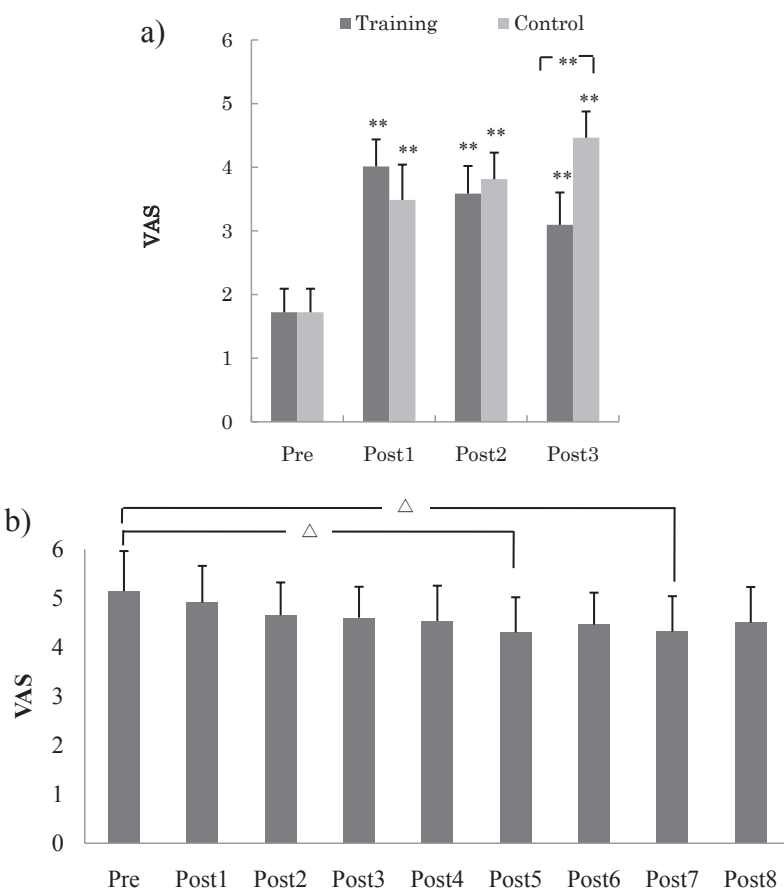

Figure 2: Time-course changes in the VAS for evaluating visual fatigue, in the measurement $1 \mathrm{a})$, in the measurement $2 \mathrm{~b})\left({ }^{* *} p<0.01, \Delta p<0.10\right)$. 


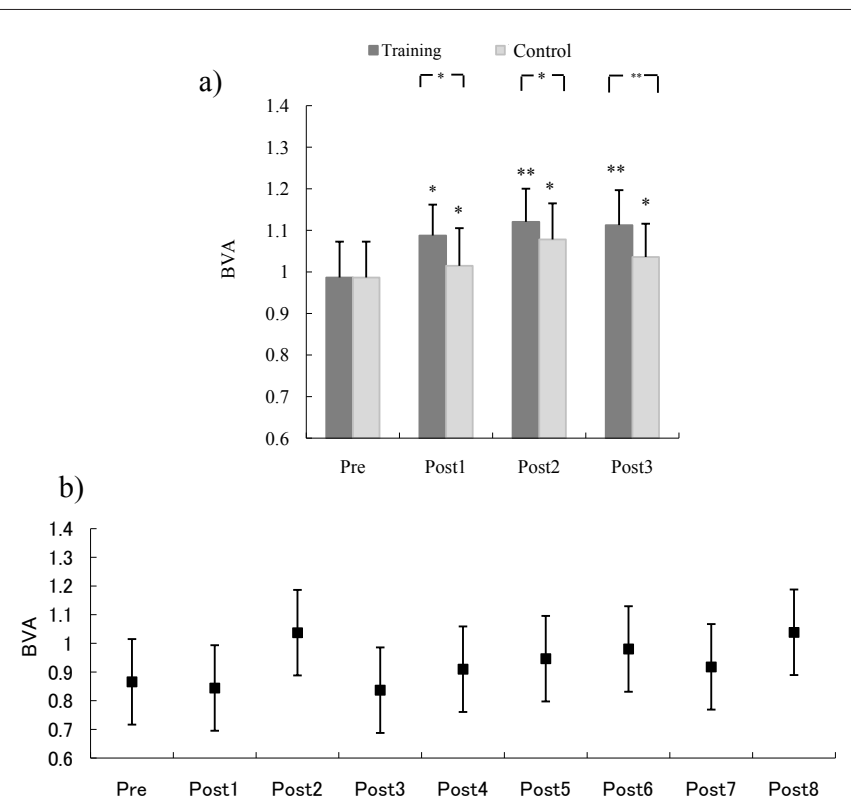

Figure 3: Time-course changes in binocular BVA at a distance, in the measurement $1 \mathrm{a}$ ), in the measurement $2 \mathrm{~b})\left({ }^{* *} \mathrm{p}<0.01,{ }^{*} \mathrm{p}<0.05\right)$.

a)
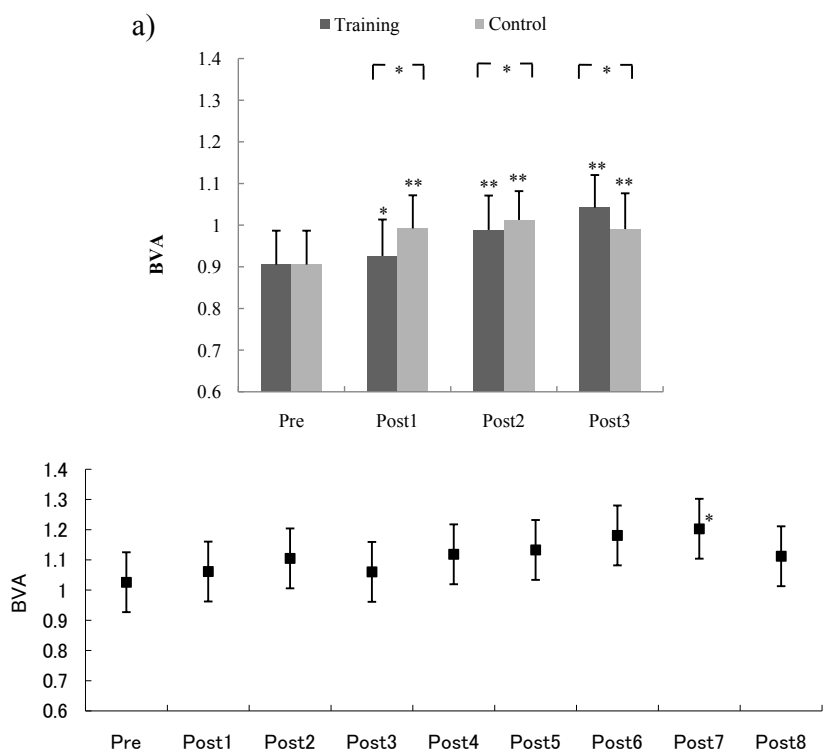

Figure 4: Time-course changes in binocular BVA from near, in the measurement $1 \mathrm{a})$, in the measurement $2 \mathrm{~b})\left({ }^{* *} p<0.01,{ }^{*} p<0.05\right)$.

increased with the viewing frequency from the $1^{\text {st }}$ to the $8^{\text {th }}$ viewing, although there was some variation. On comparison of the uncorrected near visual acuity between the Pre and after the $7^{\text {th }}$ video viewing employing the Wilcoxon signed-rank test, a significant difference was noted $(\mathrm{p}<0.05)$.

\section{Discussion}

In this study, we presented a movie using the POWER 3D method as the movie for the accommodation training, and a short-term effect of the accommodation training was investigated in visual inspection workers suffering from eye fatigue and middle-aged people. In both measurements, the motion sickness could not be induced by viewing $3 \mathrm{D}$ video clips in accordance with subjective tests, and the visual acuity was improved by continuous training.

In measurement 1 , we showed that the visual inspection workers suffered from eye fatigue after their work as shown in Figure 2a. Although the dioptric comparison between the control and the training groups showed that there was no significant difference between the values for the groups $(\mathrm{p}<0.05)$, the binocular BVA increased in 13 of the 22 visual inspection workers (59.1\%) [17].

As shown in Figure 2a-3a, the visual acuity of the control group without the accommodation training showed an improvement. The myopic tendency had increased due to the visual inspection work. Moreover, it was possible that the subjects became skilled in the vision test. However, the results obtained from the Wilcoxon signedrank test showed that the distant visual acuity in the training group had increased considerably compared to that in the control group $(\mathrm{p}<$ 0.05). As compared to the near visual acuity in the control group, that in the training group had increased significantly on day $3(\mathrm{p}<0.05)$.

The VAS in the training group had also increased significantly on day 3 as compared to that in the control group (Figure 5). There seemed to be not only visual acuity improving effect but also reduction of the visual fatigue by the accommodation training for more than 3 consecutive days.

The authors have verified a middle-term effect of accommodation training that uses the strategy of presenting a stereoscopic movie to 32 myopic youth $(20 \pm 1$ years). The movie consists of one to five balls moving back and forth in the stereoscopic sky background. At a viewing distance of $60 \mathrm{~cm}$, the stereoscopic ball is viewed to move from $30 \mathrm{~cm}$ (forward) to infinity (backward). This ball completes a round-trip movement more than 25 times in $3 \mathrm{~min}$. The uncorrected distant visual acuity increased in 17 of the 32 subjects $(53.1 \%)$ participating in this study. Although there were some variations, the visual acuity improved in the accommodation training group and not in the control group. Upon comparing the value obtained on each measurement day using the Wilcoxon signed ranks test, we found that the visual acuity on day 11 was considerably higher in the accommodation group than in the control group $(\mathrm{p}<0.05)$. This result suggests that the accommodation training using a stereoscopic movie has a cumulative positive effect on eyesight and prevents the deterioration of visual acuity. Although the myopic tendency improved slightly in the accommodation training group, there was only slight progress in the control group. These results suggested that the accommodation training using the stereoscopic movie did not deform the lens, thus not improving myopia fundamentally [18].

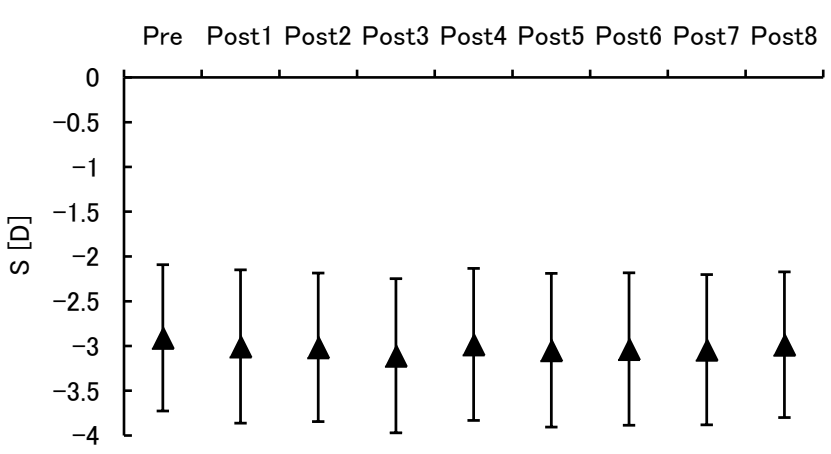

Figure 5: Time-course changes in SPH. 
Citation: Takada M, Miyao M, Satoh M, Yoshikawa K, Matsuura Y (2012) Effect of Accommodation Training on Visual Function of Visual Inspection Workers and Middle-Aged People. J Sports Med Doping Stud 2:112. doi:10.4172/2161-0673.1000112

Page 4 of 4

We assumed that these effects of the accommodation training were temporary, but the findings suggest that the continuous accommodation training will promote a ciliary-muscle-stretching effect, leading to an improvement in visual acuity. Accommodation reflex for near vision may be defined as the mechanism of working the ciliary muscle. This may also inhibit a reduction in the visual acuity.

In measurement 2 , both the binocular near and distant visual acuities were improved, suggesting that the viewing of the stereoscopic video reduced strain and increased the flexibility of the ciliary muscle, which temporarily recovered the visual acuity. In contrast, findings on objective refractometry at Pre and post viewing stereoscopic videos were compared employing the Wilcoxon signed-rank test, setting the significance level to 0.05 [19]. Changes in the spherical diopter (SPH) of individual eyes with the frequency of video viewing are shown in Figure 5. The mean SPH was about -3 diopters in both eyes, showing no significant changes with the viewing frequency. Therefore, diopteric measurements did not change in either eye. The duration of the accommodation training may have been too short to modify the eyeball (lens) structure. It was suggested that the short-term repeated use of the accommodation training increased the near visual acuity, for which the improvement and prevention of presbyopia may be expected.

\section{Conclusions}

The visual acuity-improving effect of the visual acuity recovery device utilizing stereoscopic videos was investigated in visual inspection workers suffering from eye fatigue and middle-aged subjects becoming aware of presbyopia, and the visual acuity was improved by the continuous accommodation training for a short-term period. We are planning to investigate the effect of the device employed for a prolonged period.:

\section{References}

1. Nakazawa T, Okubo Y, Suwazono Y, Kobayashi E, Komine S, et al. (2002) Association between duration of daily VDT use and subjective symptoms. Am $\mathrm{J}$ Ind Med 42: 421-426.

2. Kobayashi S (1994) Eye sight recovering apparatus. Japan Patent H6 339501.

3. Miyao M, Ishihara SY, Saito S, Kondo TA, Sakakibara H, et al. (1996) Visual accommodation and subject performance during a stereoscopic object task using liquid crystal shutters. Ergonomics 39: 1294-1309.

4. Omori M, Watanabe T, Takai J, Takada H, Miyao M (2003) An attempt at preventing asthenopia among VDT workers. Int J Occup Saf Ergon 9: 453-462.

5. Fukuda T (1991) Aging and visual fuctions. ITEJ technical report 15: 1-8.

6. Ukai K, Howarth PA (2008) Visual fatigue caused by viewing stereoscopic motion images: Background, theories, and observations. Displays 29: 106-116.

7. Kennedy RS, Lane NE, Berbaum KS, Lilienthal MG (1993) Simulator Sickness Questionnaire: An Enhanced Method for Quantifying Simulator Sickness. Int J Aviation Psychology 3: 203-220.

8. Holomes SR, Griffin MJ (2001) Correlation between heart rate and the severity of motion sickness caused by optokinetic stimulation. J Psychophysiology 15: 35-42.

9. Himi N, Koga T, Nakamura E, Kobashi M, Yamane M, et al. (2004) Differences in autonomic responses between subjects with and without nausea while watching an irregularly oscillating video. Auton Neurosci 116: 46-53.

10. Yokota Y, Aoki M, Mizuta K, Ito Y, Isu N (2005) Motion sickness susceptibility associated with visually induced postural instability and cardiac autonomic responses in healthy subjects. Acta Otolaryngol 125: 280-285.

11. Scibora LM, Villard S, Bardy B, Stoffregen TA (2007) Wider stance reduces body sway and motion sickness. in Proc. the First International Symposium on Visually Induced Motion Sickness, Fatigue, and Photosensitive Epileptic Seizures. Hong Kong 18-23.
12. Yasui R, Matsuda I, Kakeya H (2006) Combining volumetric edge display and multiview display for expression of natural 3D images. SPIE 6055: 315-323.

13. Kakeya $\mathrm{H}$ (2007) MOEVision:simple multiview display with clear floating image. SPIE 6490: 1-8.

14. Nishihira T, Tahara H (2008) Apparatus for recovering eyesight utilizing stereoscopic video and method for displaying stereoscopic video. US7 404639 B2.

15. Takada H, Fujikake K, Omori M, Hasegawa S, Watanabe T, et al. (2009) Reduction of body sway can be evaluated by sparse density during exposure to movies on Liquid Cristal Displays. IFMBE Proceedings 23: 987-991.

16. Takada H, Fujikake K, Watanabe T, Hasegawa S, Omori M, et al. (2009) A method for evaluating motion sickness induced by watching stereoscopic images on head-mounted display. SPIE 7237: 1-8.

17. Takada M, Miyao M, Shiozawa T, Matsuura Y, Omori M et al. (2010) Effect of eyesight-recovering stereoscopic movie system on visual acuity and fatigue of visual inspection workers. IADIS Visual Communications 494-497.

18. Takada H, Yamamoto T, Sugiura S, Miyao M (2009) Effect of an Eyesight Recovering Stereoscopic Movie System on Visual Acuity of middle-aged and Myopic Young People. IFMBE Proceedings 25: 331-334.

19. Sugiura A, Miyao M, Yamamoto T, Takada H (2011) Effect of strategic accommodation training by wide stereoscopic movie presentation on myopic young people of visual acuity and asthenopia. Displays 32: 219-224. 